\title{
Nutritional Counseling in Prevention of Caries - A Team Approach
}

\author{
Ankush Datta*, Geetika Datta \\ Private practice (New Delhi) \\ *Corresponding author: drankushdatta@gmail.com
}

Received October 19, 2014; Revised October 29, 2014; Accepted December 18, 2014

\begin{abstract}
Inappropriate dietary habits and unhealthy food preferences developed during childhood have lifetime implications. Therefore, it is not wise to wait until the oral or chronic disease manifests; every child's diet should be screened and evaluated at regular intervals. Unhealthy dietary habits and food choices should be identified, appropriate recommendations and referrals made to limit disease risk. Pediatricians and other primary care providers have an established role in the prevention and early identification of health problems. Providing patients with ageappropriate anticipatory guidance, nutritional counseling on caries prevention, assessment and timely referrals should be a co-ordinated team approach involving pediatricians and dentists particularly the pediatric dentists.
\end{abstract}

Keywords: caries, pediatricians, prevention

Cite This Article: Ankush Datta, and Geetika Datta, "Nutritional Counseling in Prevention of Caries - A Team Approach." International Journal of Dental Sciences and Research, vol. 2, no. 6B (2014): 31-33. doi: 10.12691/ijdsr-2-6B-9.

\section{Introduction}

Since the beginning of the twentieth century, the medical profession has recognized the importance of providing prenatal counseling and care to expectant mothers. Only recently has the dental profession become involved in this primary preventive effort. Comprehensive dental care includes consideration of the nutritional aspects of oral health and disease, besides determining other potential health risks. [1,2] Although often neglected, nutritional counseling can play an important role in his. [3] Incorporating nutritional counseling as an integral component of regular dental checkups can help dental practitioners to deliver patients with an optimum level of care. Close collaboration among members of the various health professions and community support groups (e.g., dentists, physicians, nurses, nutritionists, social workers) is important to ensure appropriate scheduling of presentations and reinforcement of concepts [4].

\section{Role in Prevention of Caries}

Often Pediatricians are the first persons to confront morbidity associated with dental caries [5] and thus have an opportunity to prevent, intervene, and, in collaboration with dental colleagues, manage this disease. The American Academy of Pediatric Dentistry(AAPD) recommends that "pediatric dentists should provide dietary counseling in conjunction with other preventive services for their patients.” [2] Preventive strategies can be differentiated into 2 distinct categories. Primary prevention involves optimization of maternal dental flora before and during colonization of the oral flora of the infant. This includes reduction in the mother's constitutionally virulent, aciduric flora and down regulation of virulence genes within the aciduric flora, decreasing the child's risk of dental decay, and is the basis for first dental visit recommendations at 1 year or earlier made by various medical and dental organizations. This mode of prevention and its adjuncts are reviewed in detail in a policy statement from the American Academy of Pediatrics, "Oral Health Risk Assessment Timing and Establishment of the Dental Home” [6]. Secondary preventive strategies are hierarchical consisting of dietary counseling, oral hygiene instructions, and judicious use of fluoride .

A detailed analysis of the Behavioral factors include oral hygiene habits, feeding practices, and dietary preferences should also be done by the dental professional which includes;

- A diet rich in fermentable carbohydrates, frequent snacking, constant use of a "sippy" cup filled with fruit juice, bottle use at sleep time, and prolonged atwill breastfeeding have all been associated with early childhood caries $[7,8,9]$.

- Poor oral hygiene practices are another marker for potential dental disease [6].

- Patients with an impaired ability to maintain oral hygiene are at higher risk; this category of patients include:

a) Infants, toddlers, and young children

b) Physically challenged and developmentally disabled individuals.

c) Children undergoing orthodontic treatment or wearing other types of intraoral appliances which are difficult to clean. 
The role of pediatrician in the case of dental caries risk assessment information can be divided into three categories Category I comprises markers of disease that are provided by the patient and parent through the history and physical examination. The presence of many of these markers can be determined by the pediatrician. Category II comprises disease markers and the single true risk factor for dental caries, the presence of Mutans Streptococci. Some of the factors in Category II can be appreciated by pediatricians, but most require dental training or technologies not likely to be present in a pediatric office. Determination of Category III markers requires the use of technologies that are not practical for clinical use at this time [8].

Pediatrician on the basis of child's medical history can provide valuable information. Prematurity and very low birth weight are associated with the presence of enamel defects, often subclinical, that can predispose teeth to caries at an early age. An increased risk also exists for children who have taken syrup-based medications on a long-term basis.

A child's dental history, which might not be well known to the pediatrician, may provide substantial information. A history of early childhood caries is one of the best indicators of future dental disease, so pediatricians should routinely evaluate the dentition for obvious carious lesions or evidence of dental restorations.

By determining risk factors, the dentist can also individualize a preventive plan with protective factors that promote oral health. Risk factors can change over time. Therefore, the dentist must assess the status periodically and make appropriate modifications (Casamassimo, 1996).

\section{Anticipatory Guidance}

Pediatricians and pediatric dentists interested in promoting good oral health should identify demographic and socioeconomic risk factors for dental caries and flag high-risk children as being in need of more intensive preventive counseling.

- Nutritional counseling. Before the eruption of teeth, parents should be taught basic information about the role of diet in promoting good oral health, and dietary factors that can lead to dental decay. Pediatricians should advise parents about appropriate foods and snacks.

- Feeding practices. Inappropriate use of nursing bottles and "sippy" cups as pacifiers should be discussed. Breast-feeding is ideal nutrition and sufficient to support optimal growth and development for about the first 4-6 months after birth. Transition to other sources of nutrients should begin at about 4-6 months of age to ensure sufficient micronutrients in the diet. The American Association of Pediatric Dentistry has endorsed the AAP's policy on breast-feeding. [7] The AAP recommends breastfeeding as the ideal method of infant feeding, with exclusive breast-feeding for the first 6 months followed by the addition of iron-enriched solid foods between 6 to 12 months of age. [10] The AAPD suggests that "ad libitum nocturnal breast-feeding should be avoided after the first primary tooth begins to erupt.” [11] Prolonged at-will breastfeeding or use of a nursing bottle at sleep times should be discouraged. Parents should be apprised of the dental effects of the prolonged use of high-sugar liquids and foods.

- Oral hygiene. The pediatrician should demonstrate methods of cleaning the oral cavity and, when erupted, the teeth. Parents should be instructed to clean the infant's mouth routinely after feedings.

- Review of medications. High-sugar medications that a child is taking long term should be identified, and parents should be cautioned to clean the child's teeth after ingestion. Physicians should consider sugar-free alternatives, if available.

- Establishing A Dental Home Pediatricians should assist parents in establishing a dental home for their children by referral to a pediatric dentist or family dentist for an initial evaluation and consultation. The American Academy of Pediatric Dentistry [6] and the American Public Health Association [12] recommend that a child's first dental visit occur within 6 months after the eruption of the first tooth, typically at 1 year of age. If a family does not have access to dental care, physicians should refer to a community dental clinic or health department for care and treatment as needed.

Recommendations for the first dental examination and to establish a "dental home" include the following :

1. Immediate referral for infants with an apparent dental problem due to trauma, disease, or developmental abnormality

2. Examination at no later than 6 months after eruption of the first tooth for infants at high risk for dental disease

3. Examination at no later than 18 months for infants not at high risk for dental disease [6].

Bright Futures-Guidelines for Health Supervision of Infants, Children, and Adolescents (Green, 1994) is a comprehensive and practical resource designed to assist health professionals and families to more effectively promote the health and well-being of all children and adolescents. It recommends an initial dental appointment at around 12 months of age to assess the infant's risk for dental disease, complete a clinical examination, provide anticipatory guidance information, and schedule a followup appointment (Nowak and Casamassimo, 1995) [13].

\section{Conclusion}

Dental caries is a diet related disease that continues to be a problem for majority of the population. Primary health care providers and dentists should thoroughly understand the relationship of diet to caries and conscientiously apply that knowledge to educate the patients in general as well as counsel special high risk individuals. [5] Most dental professionals do not incorporate nutritional counseling as a service in their practices due to time constraints, questions concerning dental insurance coverage, greater prioritization placed on dental procedures. Thus a collaborative effort is mandatory for the successful implementation of nutritional counseling in pediatric medical and dental settings. It is time to think and act together. 


\section{References}

[1] Palmer CA. Nutrition, diet and oral conditions. In: Harris NO Garcia-Godoy F, eds. Primary Preventative Dentistry. 6th ed. Upper Saddle River, NJ: Pearson Education; 2003: 419-447.

[2] Cutter CR. Oral health emphasis in dietetic internship programs. J Am Diet Assoc. 1986; 86: 84-85.

[3] Elborn S, Karp WR. The dietitian as a member of the dental health care team. J Am Diet Assoc. 1987; 87: 1062-1063.

[4] Tinanoff Norman, Palmer A. Dietary determinants of dental caries and dietary recommendations for preschool children. Journal of public health dentistry. Summer 2000; 60(3).

[5] National Institute of Dental and Craniofacial Research. Oral Health in America: A Report of the Surgeon General. Rockville, MD: US Department of Health and Human Services, National Institute of Dental and Craniofacial Research; 2000.

[6] Preventive Oral Health Intervention for Pediatricians. Pediatrics 2008; 122: 1387-1394.

[7] Boyd LD, Dwyer JT, Palmer CA. Current Concepts for the Application of Nutrition in Oral Health Promotion and Disease Prevention. Boston, Mass: Joint Project of Frances Stern Nutrition
Center, Tufts University School of Nutrition Science and Policy, Tufts University School of Medicine, Dept of Preventive Dentistry and Dept of Continuing Education; 1998.

[8] Moss ME, Zero DT: An overview of caries risk assessment, and its potential utility. J Dent Educ 1995; 59: 932.

[9] Holli BB, Calabrese RJ. Counseling. In: Communication and Education Skills for Dietetics Professionals. 3rd ed. Baltimore, Md: Williams and Wilkins; 1998: 45-58.

[10] American Academy of Pediatrics. Breast-feeding andthe use of human milk. Pediatrics. 1997; 100: 1035-1039.

[11] American Academy of Pediatric Dentistry. Policy on baby bottle tooth decay (BBTD)/Early Childhood Caries (ECC). Pediatr Dent. 2002; 24(7): 23.

[12] American Academy of Pediatric Dentistry. Policy on breastfeeding. Pediatr Dent. 2002; 24(7): 22.

[13] American Academy of Pediatrics, Bright Futures Steering Committee.Promoting oral health. In: Hagan JF, Shaw JS, DuncanPM, eds. Bright Futures: Guidelines for Health Supervision of Infants,Children, and Adolescents. 3rd ed. Elk Grove Village, IL:American Academy of Pediatrics; 2008: 155168. 